\title{
A study of prevalence of urogenital problem among menopausal women attending the OPD at tertiary care hospital in Bhopal
}

\author{
Malini Bharadwaj, Tarani Shinde*
}

Department of Obstetrics and Gynaecology, LN Medical College, Bhopal, Madhya Pradesh, India

Received: 07 January 2021

Accepted: 06 February 2021

*Correspondence:

Dr. Tarani Shinde,

E-mail: dr.tarani0302@gmail.com

Copyright: (c) the author(s), publisher and licensee Medip Academy. This is an open-access article distributed under the terms of the Creative Commons Attribution Non-Commercial License, which permits unrestricted non-commercial use, distribution, and reproduction in any medium, provided the original work is properly cited.

\begin{abstract}
Background: The interest in menopausal problems has increased in recent years, due to the expansion of human life span. Women are spending more than one-third of their lives in menopausal period. Due to this fact, estrogen deprivation can cause vaginal atrophy symptoms and vasomotor symptoms, which critically diminish quality of life (QOL) in postmenopausal women.

Methods: This study is a retrospective observational/analytical study conducted in the menopausal specialty clinic in J. K. Hospital, L. N. Medical College, Bhopal over a period of 1 year. All the women who attained menopause either naturally or surgically who presented first time to the clinic for any complaints were evaluated for the UG symptoms with the help of face to face interview.

Results: Out of the 300 women with menopausal symptoms, 182 i.e. $60.6 \%$ were married and 52\% (156) were illiterate. The most prevalent menopausal symptom in present study was vaginal dryness 240 (80\%).

Conclusions: There is association with lower educational levels. Hence efforts should be made educate women about the symptoms and provide care at all the levels of health care delivery.
\end{abstract}

Keywords: Menopause, OBG, Urogenital problem

\section{INTRODUCTION}

The interest in menopausal problems has increased in recent years, due to the expansion of human life span. Women are spending more than one-third of their lives in menopausal period. Due to this fact, estrogen deprivation can cause vaginal atrophy symptoms and vasomotor symptoms, which critically diminish quality of life (QOL) in postmenopausal women., ${ }^{1,2}$

Although climacteric symptoms, particularly vasomotor symptoms, may be transient, atrophic symptoms affecting the vagina and lower urinary tract are often progressive and frequently require treatment. ${ }^{2}$ Urogenital (UG) symptoms are one of the most common symptoms of menopause. These symptoms include vaginal dryness, itching, dyspareunia, and urinary incontinence (UI). ${ }^{3-5}$
The percentage of women seeking medical help is somewhere around $20 \%-25 \%$. the urinary and vaginal symptoms can be decreased effectively by local application of estrogenic creams..$^{6-8}$

Women with vaginal atrophy complain about pain, dryness, dyspareunia, vaginal discharge, burning sensation, decrease in vaginal lubrication leading to loss of interest in sexual activity. ${ }^{9}$

The term genitourinary syndrome of menopause (GSM) describes various menopausal symptoms and signs associated with physical changes of the vulva, vagina, and lower urinary tract. It includes urinary symptoms along with genital and sexual symptoms. ${ }^{10} \mathrm{GSM}$ is mostly underdiagnosed due to sexual embarrassment or general disregard due to associating it as a liability of natural 
aging. A study showed, $4 \%$ of women were able to attribute vulvovaginal symptoms to GSM. ${ }^{11}$ The current study was done to look for the extent of the problem in our study area.

\section{METHODS}

This study is a retrospective observational/analytical study conducted in the menopausal specialty clinic in $\mathrm{J}$. K. Hospital, L. N. Medical College, Bhopal over a period of 1 year. All the women who attained menopause either naturally or surgically who presented first time to the clinic for any complaints were evaluated for the UG symptoms with the help of face to face interview. All the patients who fulfilled the inclusion criteria attending the obstetric and gynecological outpatient department were included in the study. The total sample size was 300 postmenopausal women.

Natural menopause was defined as the cessation of menstruation for at least 1 year. Surgical menopause was defined as menopause after bilateral oophorectomy with or without hysterectomy. On follow-up visits, those women were also included who developed UG complaints which were not present at the first visit. Women with preexisting complaints before menopause and those with some medical disorders such as diabetes, stroke, or neurological problems were excluded from the study.

The interview includes questions UG symptoms assessment included vaginal dryness, vaginal discharge or infection, dyspareunia, loss of libido, urgency, burning micturition, and UI. Data were analyzed by standard statistical analytical tests.

Permission was obtained from the ethical committee and each patient after explaining the purpose of the study. The study uses face to face interview for assessing urogenital problems in menopausal women. Participants were enquired about urogenital symptoms sexual problems, bladder problems and dryness of the vagina. Socio demographic data included age, marital status, educational level, occupation and average household income was also collected.

The questions was in local language and women were interviewed face to face. They were asked question on whether or not they had experienced the urogenital symptoms. All the women were interviewed in local language.

They were explained the various urogenital symptoms and face to face communication with the women was done by trained health professional so as to make sure that right responses were received and also a proper explanation were given to women if they had doubts. All the women who fulfilled the criteria were invited to participate in the study. A written informed consent was taken from them.

\section{RESULTS}

The study had 300 patients. The mean age of menopause in this study was $52.33 \pm 4.28$ years ranging between 50 54 years (Table 1). Out of the 300 women with menopausal symptoms, 182 i.e. $60.6 \%$ were married and $52 \%$ (156) were illiterate (Table 2). The co-relation between illiteracy and presence of menopausal symptom was found to be statistically significant. On the basis of the socioeconomic status majority of the women ranging almost $79 \%$ belongs to the lower socioeconomic status. The women belonging to lower socioeconomic status were found to have more menopausal symptoms and this was statistically significant.

Table 1: Distribution of patients based on age at menopause.

\begin{tabular}{|lll|}
\hline $\begin{array}{l}\text { Age at } \\
\text { menopause }\end{array}$ & $\begin{array}{l}\text { Number of } \\
\text { patients }\end{array}$ & Percentage \\
\hline $\mathbf{4 0 - 4 4}$ & 58 & 19.3 \\
\hline $\mathbf{4 4 - 4 9}$ & 68 & 22.6 \\
\hline $\mathbf{5 0 - 5 4}$ & 116 & 38.6 \\
\hline $\mathbf{5 5 - 5 9}$ & 30 & 10 \\
\hline $\mathbf{6 0 - 6 5}$ & 28 & 9.3 \\
\hline Total & 300 & 100 \\
\hline
\end{tabular}

Table 2: Association of cases according to education and symptoms $(\mathrm{n}=300)$.

\begin{tabular}{|llll|}
\hline Education & Symptoms & Percentage \\
\hline Uneducated & Present & Absent & \\
\hline Educated & $112(92.3)$ & $12(7.7)$ & $156(52)$ \\
\hline Total & $256(85.3)$ & $32(22.2)$ & $144(48)$ \\
\hline
\end{tabular}

$\mathrm{p} \leq 0.001$

Table 3: Distribution of patients based on genitourinary symptoms in menopausal women.

\begin{tabular}{|lll|}
\hline $\begin{array}{l}\text { Menopausal } \\
\text { symptoms }\end{array}$ & $\begin{array}{l}\text { Number of } \\
\text { patients }\end{array}$ & Percentage \\
\hline Vaginal dryness & 240 & 80 \\
\hline Vaginal itching & 228 & 76 \\
\hline Vaginal discharge & 200 & 66.7 \\
\hline Dysuria & 180 & 60 \\
\hline Burning micturition & 160 & 53.3 \\
\hline Dyspareunia & 170 & 56.7 \\
\hline Frequency & 140 & 46.7 \\
\hline Loss of libido & 114 & 38 \\
\hline $\begin{array}{l}\text { Urinary } \\
\text { incontinence }\end{array}$ & 90 & 30 \\
\hline Urgency & 65 & 21.6 \\
\hline
\end{tabular}

The most prevalent menopausal symptom in present study was vaginal dryness $240(80 \%)$, followed by vaginal itching $(76 \%)$. This was followed by vaginal discharge $200(66.7 \%)$, dysuria180 (60\%), burning 
micturition $160 \quad(53.3 \%)$, dyspareunia $170 \quad(56.7 \%)$, frequency $140(46.7 \%)$, loss of libido $114(38 \%)$, urinary incontinence 90 (30\%), urgency 65 (21.6\%) (Table 3).

\section{DISCUSSION}

Ageing is an in inevitable phenomenon and with it are associated certain conditions which affects quality of life. Menopause is one such reality of life. Menopause is characterised by an oestrogen deficient state and as many organs of the body are sensitive to oestrogen, a decrease in oestrogen level gives rise to a number of physical, psychological and sexual changes. The frequency of symptoms varies over time. Some happen frequently in the perimenopause and decrease over time, while others increase progressively from perimenopause to post menopause and become more severe towards the end of life.

The mean age at menopause in our study was $52.33 \pm 4.28$ years which is somewhat earlier when compared to western counter parts which is around 51.14 \pm 2.11 years worldwide. $^{12}$ However it is slightly higher than the average age of menopause as found by Indian menopause society which is around 47.5 years.

In various other studies, the mean age of menopause fell between 49.4 to 51.1 years. The studies done in Thailand (48.7 years) Singapore (49.1 years) and other studies on Asian women our findings also show similar age of menopause. ${ }^{13,14}$ The assessment tool which we used in present study was personal face to face interview of women by health care professional.

In our study we did a face to face interaction with the respondents. A health-personnel explained the symptoms and its severity to the respondents and how avoiding and hiding this problems can affect the quality of life. The aim of involving a health personal and a face to face interaction was to minimize the reporting error and educate the postmenopausal women about urogenital problem and how timely treatment can improve quality of life.

In present study, out of the 300 respondents with either one or multiple menopausal symptoms, 156 had no formal education. In various previous studies it was shown that urogenital symptoms in menopausal were inversely related with educational level.

In a study by Lee et al, it was shown that subject's income was related to there educational level and low income could be one of the risk factors for more severe urogenital symptoms. ${ }^{15}$ However in a study by Joseph et al they found educated women having more menopausal symptoms. ${ }^{16}$ Similarly, the respondents belonging to lower socio-economic strata were found to have more menopausal symptoms and this difference in proportion we found to be statistically significant.
It was shown by Lee et al in his study that subject's income was related to there educational level and low income could be one of the risk factors for more severe urogenital symptoms. ${ }^{15}$

The most common symptom which we encountered in our study was vaginal dryness $(80 \%)$. This finding was consistent with many other studies where the same problem was found to be prevalent. In a study by Cheng et al and Hafiz et al also found dysuria as a predominant urinary complaint. Hemmat et al found that Urinary incontinence (UI) is the debilitating condition with impact on physical and psychological aspect of life with consequent effect on the quality of life (QoL). ${ }^{17,18}$

Differences in norms and traditions, culture, sources of food and others styles of life play an important role in the prevalence of menopause symptom. In a study by Pal et al, the prevalence of physical and mental exhaustion was found to be much more higher $(86 \%){ }^{19}$ The urogenital problems like sexual problems $(20 \%)$ and dryness of vagina were found to be less prevalent and if present then the symptoms were mild. It is probably because women are more hesitant on sharing their genitourinary complaints.

Pal et al found in their study that bladder problem (56\%) and vaginal dryness $(53.3 \%)$ were much prevalent. ${ }^{19}$ Thus, the variation in the prevalence of different menopausal symptoms at different places is highly dependent on the tradition, culture, sources of food and other styles of life. Thus, we have to understand that menopause is a stage of reproductive life cycle of woman. Menopause is a bio psychological phenomenon and is a natural aging process which signals a decline in body function. The body undergoes various physiological changes due to the estrogen deficiency. This needs the combined medical and psychosocial support. Hence, the women can have the strength to overcome the severity of changes which affects the wellbeing of women.

There were several limitations in our study. Although attempts were made to ensure that the data collection was appropriate, however when women were asked to provide some retrospective information such as menopausal symptoms experienced in the preceding one month, last menstruation, recall bias was unavoidable, especially for some elderly women.

\section{CONCLUSION}

This study on menopausal women of Northern India between the ages 40-65 years was done using face to face interview. The mean age of menopause in our study was $52.33 \pm 4.28$ years.

The educational level and socioeconomic status level had inverse relationship with the menopausal symptoms showing that respondents of lower socio- economic strata and uneducated were more prone to experience 
menopausal symptoms. This relationship was found to be statistically significant. The most common symptom reported in present study was vaginal dryness $240(80 \%)$, followed by vaginal itching $(76 \%)$.

There is association with lower educational levels. Hence efforts should be made to educate women about the symptoms and provide care at all the levels of health care delivery.

\section{Funding: No funding sources}

Conflict of interest: None declared

Ethical approval: The study was approved by the Institutional Ethics Committee

\section{REFERENCES}

1. Wiklund I. Methods of assessing the impact of climacteric complaints on quality of life. Maturitas. 1998;29(1):41-50.

2. Recommendations for the management of postmenopausal vaginal atrophy. Sturdee DW, Panay N, International Menopause Society Writing Group. Climacteric. 2010;13(6):509-22.

3. Sakondhavat C, Choosuwan C, Kaewrudee S, Soontrapa S, Louanka K. Prevalence and risk factors of urinary incontinence in Khon Kaen menopausal women. J Med Assoc Thai. 2007;90:2553-8.

4. Nappi RE, Lachowsky M. Menopause and sexuality: Prevalence of symptoms and impact on quality of life. Maturitas. 2009;63:138-41.

5. Bozkurt N, Ozkan S, Korucuoğlu U, Onan A, Aksakal N, Ilhan M, et al. Urogenital symptoms of postmenopausal women in Turkey. Menopause. 2007; 14:150-6.

6. Nappi RE, Davis SR. The use of hormone therapy for the maintenance of urogynecological and sexual health post WHI. Climacteric. 2012;15:267-74.

7. Huang AJ, Luft J, Grady D, Kuppermann M. The day-to-day impact of urogenital aging: perspectives from racially/ethnically diverse women. J Gen Intren Med. 2009;14:123-30.

8. Archer DF. Efficacy and tolerability of local estrogen therapy for urogenital atrophy. Menopause. 2010;17:194-203.

9. Schaffer J, Fantl A. Urogenital effects of the menopause. Balliere's Clin Obstet Gynaecol. 1996;10:401-17.
10. Portman DJ, Gass ML. Genitourinary syndrome of menopause: new terminology for vulvovaginal atrophy from the International Society for the Study of Women's Sexual Health and the North American Menopause Society. Menopause. 2014;21:1063-8.

11. Gandhi J, Chen A, Dagur G, Suh Y, Smith N, Cali B, Khan SA. Genitourinary syndrome of menopause: an overview of clinical manifestations, pathophysiology, etiology, evaluation, and management. Am J Obstet Gynecol. 2016;215(6):704-11.

12. Rahman S, Salehin F, Iqbal A. Menopausal symptoms assessment among middle age women in Kushtia, Bangladesh. BMC Research Notes. 2011;4(1):188

13. Peeyananjarassri K, Cheewadhanaraks S, Hubbard M, Manga ZR, Manocha R, Eden J. Menopausal Symptoms in a hospital-based sample of women in southern Thailand. Climacteric. 2006;9:23-9.

14. Boulet MJ, Oddens BJ, Lehert P, Verme HM, Visser AP. Climacteric and menopause in seven South-east Asian countries. Maturitas. 1994;19:157-76.

15. Lee Y, Kim H. Relationship between menopausal symptoms, depression, and exercise in middle aged women. Int J Nurs Studies. 2008;45:1816-22.

16. Joseph N, Nagaraj K, Saralaya V, Nelliyanil M, Rao PJ. Assessment of menopausal symptoms among women attending various outreach clinics in South Canara District of India. J Mid-Life Health. 2014;5(2):84-90.

17. Cheng MH, Wang SJ, Wang PH, Fuh JL. Attitudes towards menopause among middle aged women: a community survey in an island of Taiwan. Maturitas. 2005;52:348-55.

18. Hafiz I, Liu J, Eden J. A quantitative analysis of the menopause experience o Indian women living in Sydney. Aust NZJ Obstet Gynaecol. 2007;47:32934.

19. Pal A, Hande D, Khatri S. Assessment of menopausal symptoms in perimenopause and post menopause women above 40 years in rural area Maharashtra (India). Int J Healthcare Biomed Res. 2013;1(3):166-74.

Cite this article as: Bharadwaj $\mathrm{M}$, Shinde $\mathrm{T}$. A study of prevalence of urogenital problem among menopausal women attending the OPD at tertiary care hospital in Bhopal. Int J Reprod Contracept Obstet Gynecol 2021;10:1062-5. 